\title{
A computed tomography (CT)-derived radiomics approach for predicting primary co-mutations involving TP53 and epidermal growth factor receptor (EGFR) in patients with advanced lung adenocarcinomas (LUAD)
}

\author{
Ying Zhu ${ }^{1 \#}$, Yu-Biao Guo ${ }^{2 \#}$, Di Xu ${ }^{3 \#}$, Jing Zhang ${ }^{2}$, Zhen-Guo Liu ${ }^{4}, \mathrm{Xi} \mathrm{Wu}^{1}$, Xiao-Yu Yang ${ }^{1}$, \\ Dan-Dan Chang ${ }^{1}$, Min $\mathrm{Xu}^{5}$, Jing Yan ${ }^{5}$, Zun-Fu Ke ${ }^{6}$, Shi-Ting Feng ${ }^{1}$, Yang-Li Liu ${ }^{2}$ \\ ${ }^{1}$ Department of Radiology, The First Affiliated Hospital of Sun Yat-sen University, Guangzhou, China; ${ }^{2}$ Division of Pulmonary and Critical Care \\ Medicine, The First Affiliated Hospital of Sun Yat-sen University, Guangzhou, China; ${ }^{3}$ Department of Thoracic Surgery, The Central Hospital of \\ Wuhan, Tongji Medical College, Huazhong University of Science and Technology, Wuhan, China; ${ }^{4}$ Department of Thoracic Surgery, The First \\ Affiliated Hospital, Sun Yat-sen University, Guangzhou, China; ${ }^{5}$ Scientific Collaboration, CT-MR Division, Canon Medical System (China), Beijing, \\ China; ${ }^{6}$ Department of Pathology, The First Affiliated Hospital, Sun Yat-sen University, Guangzhou, China \\ Contributions: (I) Conception and design: Y Zhu, YL Liu, ST Feng, YB Guo, ZF Ke; (II) Administrative support: ST Feng, ZF Ke, YB Guo; (III) \\ Provision of study materials or patients: ST Feng, YB Guo, ZF Ke; (IV) Collection and assembly of data: Y Zhu, YL Liu, M Xu, J Yan, D Xu, ZG \\ Liu, J Zhang; (V) Data analysis and interpretation: Y Zhu, M Xu, J Yan; (VI) Manuscript writing: All authors; (VII) Final approval of manuscript: All \\ authors. \\ "These authors contributed equally to this work. \\ Correspondence to: Yang-Li Liu. Division of Pulmonary and Critical Care Medicine, The First Affiliated Hospital of Sun Yat-sen University, \\ Guangzhou 510080, China Email: liuyli3@mail.sysu.edu.cn; Shi-Ting Feng. Department of Radiology, The First Affiliated Hospital of Sun Yat-sen \\ University, Guangzhou 510080, China. Email: fengsht@mail.sysu.edu.cn; Zun-Fu Ke. Department of Pathology \& Institution of Precision Medicine, \\ The First Affiliated Hospital, Sun Yat-sen University, Guangzhou 510080, China. Email: kezunfu@mail.sysu.edu.cn.
}

Background: Epidermal growth factor receptor (EGFR) co-mutated with TP53 could reduce responsiveness to tyrosine kinase inhibitors (TKIs) and worsen patients' prognosis compared to TP53 wild type patients in EGFR-mutated lung adenocarcinomas (LUAD). To identify this genetically unique subset prior to treatment through computed tomography (CT) images had not been reported yet.

Methods: Stage III and IV LUAD with known mutation status of EGFR and TP53 from The First Affiliated Hospital of Sun Yat-sen University (May 1, 2017 to June 1, 2020) were collected. Characteristics of pretreatment enhanced-CT images were analyzed. One-versus-one was used as the multiclass classification strategy to distinguish the three subtypes of co-mutations: $E G F R^{+} \& T P 53^{+}, E G F R^{+} \& T P 53^{-}, E G F R^{-}$. The clinical model, semantic model, radiomics model and integrated model were built. Area under the receiveroperating characteristic curves (AUCs) were used to evaluate the prediction efficacy.

Results: A total of 199 patients were enrolled, including 83 (42\%) cases of $E G F R^{-}, 55$ (28\%) cases of $E G F R^{+} \& T P 53^{+}, 61(31 \%)$ cases of $E G F R^{+} \& T P 53^{-}$. Among the four different models, the integrated model displayed the best performance for all the three subtypes of co-mutations: $E G F R^{-}$(AUC, 0.857 ; accuracy, 0.817; sensitivity, 0.998; specificity, 0.663), EGFR \& TP53 (AUC, 0.791; accuracy, 0.758; sensitivity, 0.762; specificity, 0.783), EGFR \& TP53- (AUC, 0.761; accuracy, 0.813; sensitivity, 0.594; specificity, 0.977). The radiomics model was slightly inferior to the integrated model. The results for the clinical and the semantic models were dissatisfactory, with AUCs less than 0.700 for all the three subtypes.

Conclusions: CT imaging based artificial intelligence (AI) is expected to distinguish co-mutation status involving TP53 and EGFR. The proposed integrated model may serve as an important alternative marker for preselecting patients who will be adaptable to and sensitive to TKIs.

Keywords: TP53; epidermal growth factor receptor (EGFR); radiomics; tomography, X-ray computed; lung adenocarcinoma (LUAD) 
Submitted Sep 17, 2020. Accepted for publication Jan 17, 2021.

doi: 10.21037/atm-20-6473

View this article at: http://dx.doi.org/10.21037/atm-20-6473

\section{Introduction}

Lung cancer remains the most common malignancy and the leading cause of cancer-related mortality worldwide (1). In East Asia, approximately one third of all lung cancer patients are never-smokers and predominantly with female gender (2-5). These patients are more often diagnosed with adenocarcinoma and epidermal growth factor receptor (EGFR) activating mutations $(2,4,6,7)$. Patients with this genotype usually confer exquisite sensitivity to tyrosine kinase inhibitors (TKIs) $(8,9)$, and EGFR mutation has become the most important molecular marker for TKI therapy selection $(9,10)$. However, approximately $20-30 \%$ non-small cell lung cancer (NSCLC) patients harboring EGFR mutation are primary resistance to TKIs (11) and not all the patients show equal response to TKIs. Recently, some studies found that multiple primary driver gene mutations, such as EGFR and TP53, could affect patients' prognosis and response to TKIs $(12,13)$. They deem that identifying mutation types in different combinations can help to select best responders to target therapy. Primary multiple mutations may be an important factor that affects curative efficacy of TKI in NSCLC patients.

TP53, encoding the p53 protein known as a tumor suppressor gene in preventing and suppressing of abnormal cell growth, is the most frequently mutated gene in NSCLC, with mutation rates up to approximately $40 \%$ in lung adenocarcinoma (LUAD) (14-16). So far, there are a series of clinical studies $(12,13)$ focused on primary overlapping mutations involving TP53. They found that, in EGFR-mutated NSCLC patients treated with TKIs, EGFR co-mutated with TP53 could reduce responsiveness to TKIs and worsen patients' prognosis compared to TP53 wild type patients, with almost a fourfold risk of disease progression (12). Primary TP53 overlapping mutation may play a potential role in TKI resistance and act as an important factor in determining TKI sensitivity. Thus, to identify this genetically unique subset of lung cancer patients prior to treatment is of great clinical significance.

Tissue biopsy based mutational sequencing have become the gold standard of driver-gene mutation detection. However, it still has some shortcomings with regards to the situation of overlapping mutations. First, gene types depend on the scale of the testing panel. In some institutes, TP53 may not be routinely detected and large panel sequencing is a heavy financial burden for patients in the developing countries. In addition, intolerance of repeated biopsies and difficulty of accessing tissue samples limits its applicability and impedes dynamic molecular monitoring. Therefore, to identify an alternative tool to predict TP53 co-mutation status in EGFR-mutated LUAD is necessary.

Computed tomography (CT) as a routinely used technique has been wildly studied in lung cancer diagnosis and therapeutic effect evaluation. Recently, with the rapid development of artificial intelligence technique in the field of medical imaging, CT derived imaging features have been reported to be a noninvasive biomarker to predict gene expression patterns in lung cancer patients $(17,18)$, and EGFR is the most commonly studied gene and the method showed predictive power (19-21). Therefore, it is feasible to predict overlapping mutations based on CT features.

Based on previous studies and considering the impact of co-mutation status of TP53 on therapeutic efficacy of TKIs in EGFR-mutated LUAD, we aimed to noninvasively identify the genetically unique subsets concerned $E G F R$ and TP53, to help preselect the best responders to TKIs via pretreatment CT images. To the best of our knowledge, this approach has not been previously reported. We present the following article in accordance with the STARD reporting checklist (available at http://dx.doi.org/10.21037/atm-20-6473).

\section{Methods}

\section{Coborts and clinical characteristics}

Advanced LUAD [American Joint Committee on Cancer (AJCC) stage III and IV] patients with known mutation status of EGFR and TP53 were collected from The First Affiliated Hospital of Sun Yat-sen University (May 1, 2017 to June 1, 2020). This project was approved by the Ethics Committee and Institutional Review Board of Sun Yat-sen University \{No.[2013]C-084\}. Informed consent was waived. This study conformed to the provisions of the Declaration of Helsinki (as revised in 2013) (available at https://www.wma.net/wp-content/uploads/2016/11/ DoH-Oct2013-JAMA.pdf). The study patients were confirmed by biopsy of the original tumor tissue, as well as immunohistochemistry. Metastasis were evaluated by 


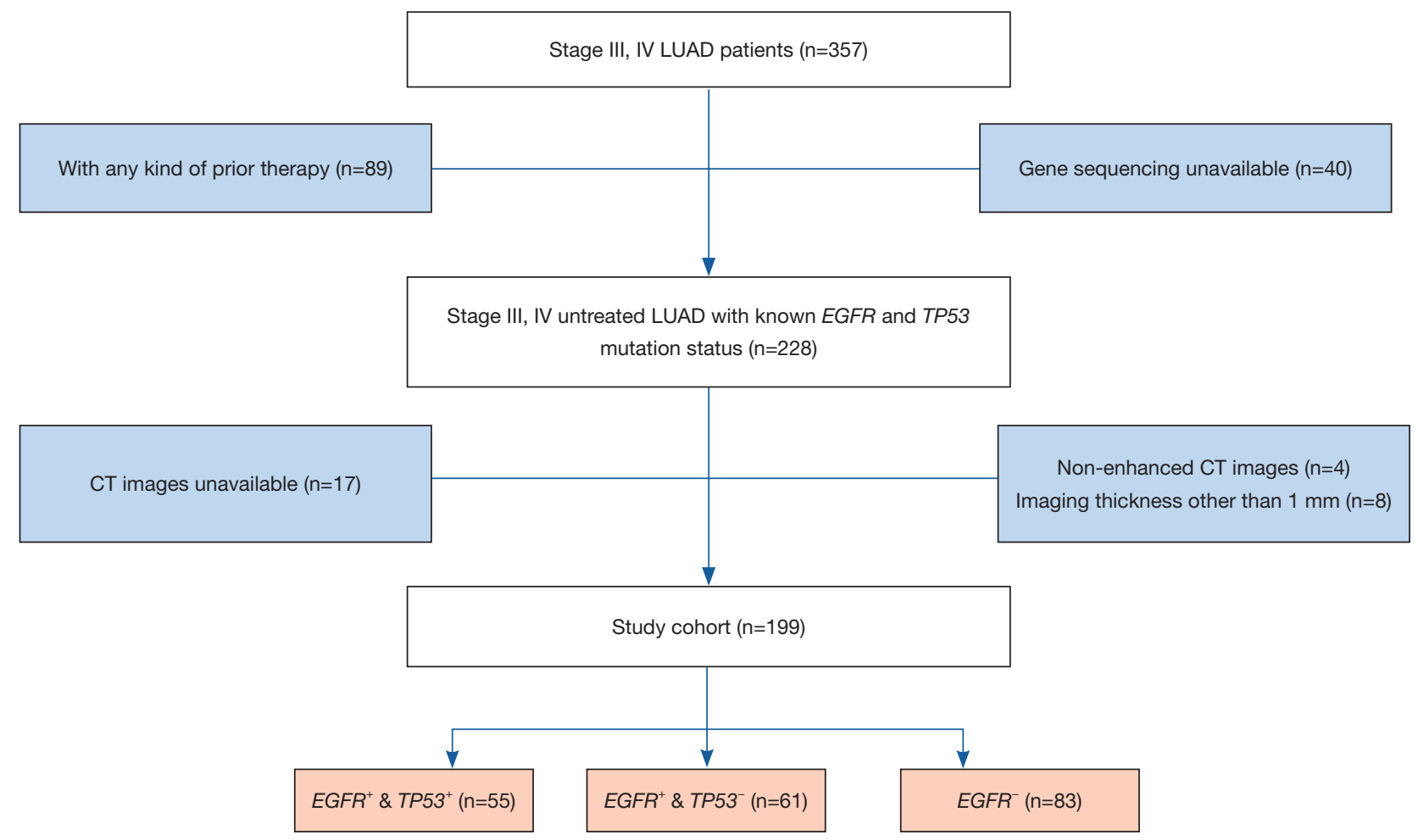

Figure 1 Flowchart of patient enrollment. LUAD, lung adenocarcinomas; CT, computed tomography; EGFR, epidermal growth factor receptor.

contemporaneous multi-site $\mathrm{CT} / \mathrm{magnetic}$ resonance imaging (MRI) or positron emission tomography (PET)CT scans of the whole body. All the enrolled patients were first-visit and prior to treatment. CT images were acquired within one week after admission and before therapy. Patients previously treated were excluded in this study. Patient enrollment algorithm is shown in detail in Figure 1. Cohort clinical characteristics are demonstrated in Table 1. Patients were categorized into three subtypes according to the mutation status of EGFR and TP53: EGFR positive combined with $T P 53$ positive $\left(E G F R^{+} \& T P 53^{+}\right)$; $E G F R$ positive combined with $T P 53$ negative $\left(E G F R^{+}\right.$\& TP53 $3^{-}$; EGFR negative $\left(E G F R^{-}\right)$, including $E G F R$ negative combined with TP53 positive or TP53 negative. The aim of this study is to prescreen the potential best responders to TKI therapy which is not recommended for EGFR negative patients. Therefore, TP53 overlapping mutation analysis is not performed in EGFR negative group.

\section{Next-generation sequencing (NGS) for gene status}

Archival tissue from 199 patients was adequate for assessment of genetic analyses including TP53, EGFR, etc. mutational status by NGS. Genomic profiling was performed by using a commercially available capture based targeted sequencing panel (Burning Rock Biotech Ltd., Guangzhou, China), targeting at least 13 genes and spanning $1.44 \mathrm{MB}$ of human genomic regions. The mutations found were confirmed by a second, independent analysis.

\section{Scan protocol}

All preoperative enhanced chest CT images were obtained with multidetector CT scanners (Aquilion 64, Canon Medical Systems, Otawara, Japan) during inspiration. Scan parameters: tube voltage of $120 \mathrm{kVp}$; maximum of $500 \mathrm{~mA}$ with automatic tube current modulation. Axial thinsection CT images of the whole lung were reconstructed with a section thickness of $1.0 \mathrm{~mm}$ at the same increment. Iopromide (300 mg I/mL, Schering Pharmaceutical Ltd.) was used as the contrast agent for enhanced scanning protocol, and $80-100 \mathrm{~mL}$ was injected at $3-4 \mathrm{~mL} / \mathrm{s}$ flow rate. In order to ensure the uniformity of image features 
Table 1 Clinicopathological characteristics of patients with advanced LUAD

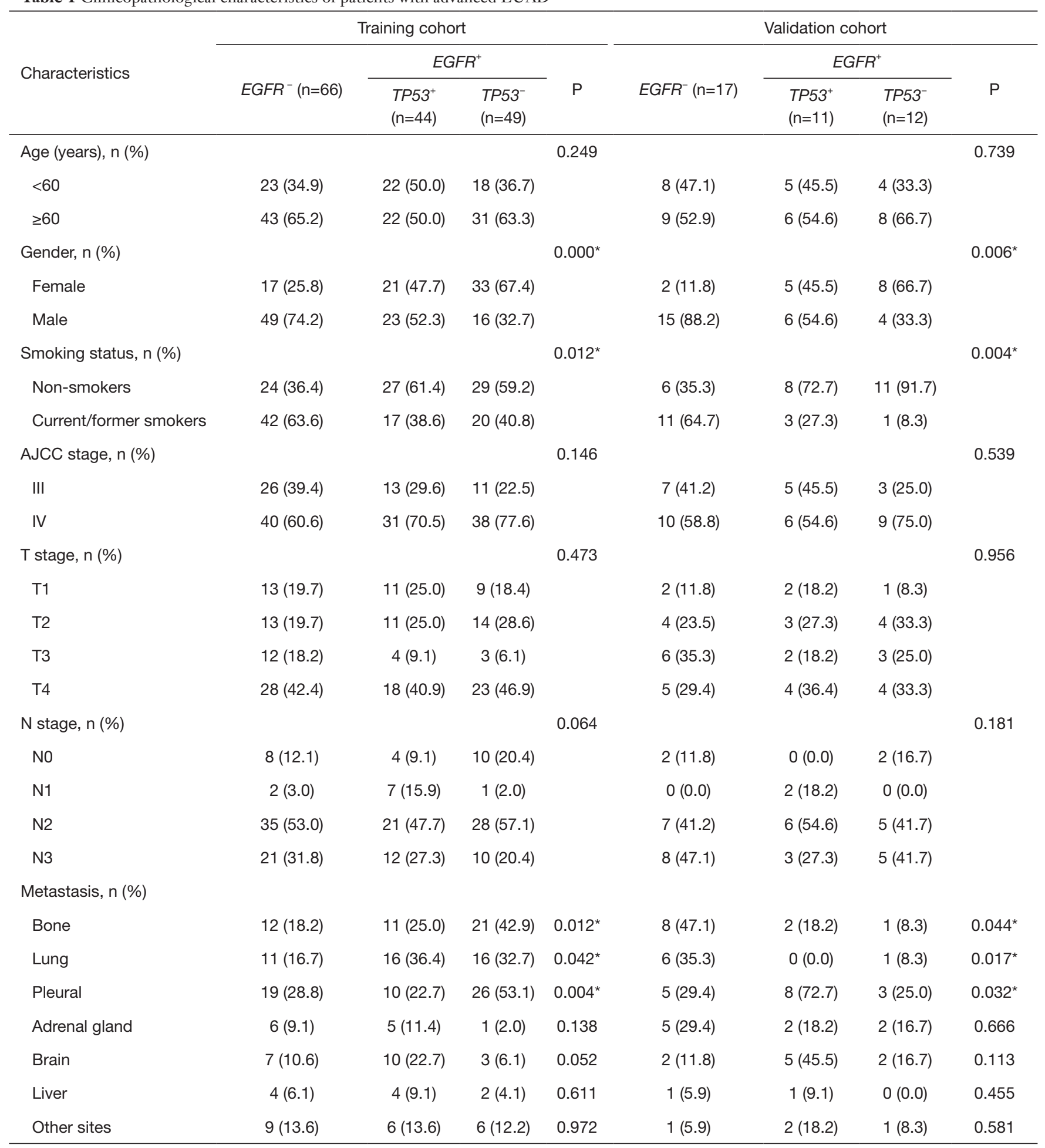

*, P value <0.05. AJCC, American Joint Committee on Cancer; LUAD, lung adenocarcinomas; EGFR, epidermal growth factor receptor. 
extracted by machine learning and to avoid feature extraction bias, patients with imaging thickness other than $1 \mathrm{~mm}$ were excluded in our study, as shown in Figure 1.

\section{Semantic CT characteristics}

Enhanced chest CT images were acquired within one week prior to treatment and the CT imaging features were evaluated by three experienced chest radiologists (XY Yang, DD Chang and $\mathrm{X} \mathrm{Wu}$, with experience of 21, 13 and 25 years respectively) through PACS reading workstation blinded to gene mutation status to control potential bias. The order of all patients was disrupted during analysis. Consensus was reached when the radiologists disagreed.

Semantic CT imaging characteristics for evaluation included (Table 2) maximum diameter; location; morphology; contour; shape; enhanced attenuation [Hounsfield units (HU)]; presence of ground-glass opacity (GGO), peri-lesion emphysema, air bronchogram, bubble-like lucency, lobulation, spiculation, pleural tag, vascular convergence, vascular involvement, homogenous attenuation, pleural effusion.

Imaging features designation: maximum diameter indicated maximal axial size $(\mathrm{mm})$; axial location were classified as inner, middle or peripheral region of the lung lobe; presence of GGO indicated GGO component surrounded or within the tumor; peri-lesion emphysema indicated concurrent emphysema of any cause; bubblelike lucency indicated the presence of air in the tumor; homogenous attenuation indicated the density of the lesion was homogenous after contrast administration and without definite necrosis; vascular involvement indicated vessels were narrowed, occluded, or encased by tumor tissue.

\section{Lesion segmentation and radiomics features extraction}

All enhanced CT images were manually segmented with an open-source software ITK-SNAP (http://www.itksnap. org/pmwiki/pmwiki.php) to obtain whole lesion's threedimensional volume segmentation of interest (VOI), which will be used for further feature extraction. DICOM data from two hospitals were blinded together and outlined by a chest radiologist (DD Chang) with 13 years of experiences, and then validated by a senior chest radiologist (X Wu) with 25-year experience.

The flow chart of radiomics model building was illustrated in Figure 2. Radiomic features were extracted with an open-source python platform Pyradiomics (version 2.1.2, https://pyradiomics.readthedocs.io/en/latest/\#). Features used in this study include the following three classes: (I) shaped-based features (14 features): descriptions of three dimensional size and shape of VOI; (II) first order statistics features (18 features): distribution of voxel intensities within the image region from gray-level histogram of $\mathrm{HU}$; (III) texture features in total 68 features including the gray-level co-occurrence matrix (GLCM, 22 features), gray level run length matrix (GLRLM, 16 features), gray level size zone matrix (GLSZM, 16 features) and gray level dependence matrix (GLDM, 14 features). Besides the original image, 15 filtered images were also generated for feature extraction, including wavelet transform filter (eight decompositions with low and high frequencies), Laplacian of Gaussian filter over three-sigma levels (1.0, 3.0, 5.0); square filter; square root filter; logarithm filter; gradient filter. All the feature classes, with the exception of shape, were calculated on the original and filtered images. Therefore, in this study, $(18+68+14)+(18+68) \times 15=1,390$ features were statistically analyzed.

\section{Prediction models and workflow}

All the patients were randomly split into training (80\%) and testing set $(20 \%)$. And all feature selections, classifiers establishment were based by the data in the training dataset to ensure independence from testing dataset.

Since the gene status of LUAD were classified into three groups, traditional machine learning based classifiers, including support vector machine (SVM), k-nearest neighbors $(\mathrm{KN})$, random forest (RF), decision tree (DT), logistic regression (LR), were applied to build multiclass models. The performance was compared by using 5 -fold cross-validation in the training cohort, with the best one being selected. One-versus-one was used here as the multiclass classification strategy, where the problem consists in using many binary classifiers to discriminate between each pair of classes, then the final result was predicted by the combination of the outputs of these base classifiers. In this study, these 3 base binary classifiers were: $\left(E G F R^{+} \&\right.$ $\left.T P 53^{+}\right)$vs. $\left(E G F R^{+} \& T P 53^{-}\right) ;\left(E G F R^{+} \& T P 53^{+}\right)$vs. $\left(E G F R^{-}\right)$; $\left(E G F R^{+} \& T P 53^{-}\right) v s .\left(E G F R^{-}\right)$. The flowchart of feature selection and model building was shown in Figure 2. Four types of models were built for the comparison in classifiers: (I) clinical features only, represented by clinical model; (II) semantic features only, represented by semantic model; (III) radiomics features only, represented by radiomics model; (IV) clinical \& semantic \& radiomics features, represented 
Table 2 CT Characteristics of patients with advanced LUAD

\begin{tabular}{|c|c|c|c|c|c|c|c|c|}
\hline \multirow{3}{*}{ Characteristics } & \multicolumn{4}{|c|}{ Training cohort } & \multicolumn{4}{|c|}{ Validation cohort } \\
\hline & \multirow[b]{2}{*}{$E G F R^{-}(\mathrm{n}=66)$} & \multicolumn{2}{|c|}{$E G F R^{+}$} & \multirow[b]{2}{*}{$\mathrm{P}$} & \multirow[b]{2}{*}{$E G F R^{-}(\mathrm{n}=17)$} & \multicolumn{2}{|c|}{$E G F R^{+}$} & \multirow[b]{2}{*}{$\mathrm{P}$} \\
\hline & & $\begin{array}{l}\text { TP53 } \\
(n=44)\end{array}$ & $\begin{array}{l}\text { TP53- } \\
(\mathrm{n}=49)\end{array}$ & & & $\begin{array}{l}\text { TP53 }^{+} \\
(n=11)\end{array}$ & $\begin{array}{l}\text { TP53 } \\
(\mathrm{n}=12)\end{array}$ & \\
\hline $\begin{array}{l}\text { Maximum diameter }(\mathrm{mm}) \text {, } \\
\text { median (IQR) }\end{array}$ & $38.8(23.4,51.0)$ & $\begin{array}{c}34.7(20.3 \\
41.0)\end{array}$ & $\begin{array}{l}41.9(26.0 \\
50.1)\end{array}$ & 0.124 & $39.4(21.0,62.6)$ & $\begin{array}{c}42.4(28.3 \\
47.1)\end{array}$ & $\begin{array}{c}43.3(29.3 \\
56.5)\end{array}$ & 0.259 \\
\hline Axial location, n (\%) & & & & 0.107 & & & & 0.863 \\
\hline Inner & $33(50.0)$ & $19(43.2)$ & $27(55.1)$ & & $7(41.2)$ & $4(36.4)$ & $5(41.7)$ & \\
\hline Middle & $21(31.8)$ & 8 (18.2) & $12(24.5)$ & & $6(35.3)$ & $3(27.3)$ & $5(41.7)$ & \\
\hline Peripheral & $12(18.2)$ & $17(38.6)$ & $10(20.4)$ & & $4(23.5)$ & $4(36.4)$ & $2(16.7)$ & \\
\hline Lobar location, n (\%) & & & & 0.896 & & & & 0.916 \\
\hline RUL & $14(21.2)$ & $13(29.6)$ & $14(28.6)$ & & $4(23.5)$ & $4(36.4)$ & $5(41.7)$ & \\
\hline RML & $8(12.1)$ & $6(13.6)$ & 8 (16.3) & & $2(11.8)$ & $1(9.1)$ & $1(8.3)$ & \\
\hline RLL & $19(28.8)$ & $8(18.2)$ & $10(20.4)$ & & $4(23.5)$ & $3(27.3)$ & $2(16.7)$ & \\
\hline LUL & $15(22.7)$ & $10(22.7)$ & $12(24.5)$ & & $5(29.4)$ & $3(27.3)$ & $3(25.0)$ & \\
\hline LLL & $10(15.2)$ & $7(15.9)$ & $5(10.2)$ & & $2(11.8)$ & $0(0.0)$ & $1(8.3)$ & \\
\hline Morphology, n (\%) & & & & 0.339 & & & & 0.916 \\
\hline Nodule $(<3 \mathrm{~cm})$ & $29(43.9)$ & $15(34.1)$ & $24(49.0)$ & & $4(23.5)$ & $2(18.2)$ & $3(25.0)$ & \\
\hline Mass $(\geq 3 \mathrm{~cm})$ & $37(56.1)$ & $29(65.9)$ & $25(51.0)$ & & $13(76.5)$ & $9(81.8)$ & $9(75.0)$ & \\
\hline Contour, n (\%) & & & & 0.399 & & & & 0.575 \\
\hline Smooth & $37(56.1)$ & $20(45.5)$ & $22(44.9)$ & & $8(47.1)$ & $6(54.6)$ & 4 (33.3) & \\
\hline Unsmooth & $29(43.9)$ & $24(54.6)$ & $27(55.1)$ & & $9(53.0)$ & $5(45.5)$ & $8(66.7)$ & \\
\hline Shape, n (\%) & & & & 0.982 & & & & 0.208 \\
\hline Round/oval & $41(62.1)$ & $27(61.4)$ & $31(63.3)$ & & $14(82.4)$ & $6(54.6)$ & 7 (58.3) & \\
\hline Irregular & $25(37.9)$ & $17(38.6)$ & $18(36.7)$ & & $3(17.7)$ & $5(45.5)$ & $5(41.7)$ & \\
\hline Presence of GGO, n (\%) & $8(12.1)$ & $5(11.4)$ & $15(30.6)$ & $0.016^{*}$ & $3(17.7)$ & $1(9.1)$ & $5(41.7)$ & 0.149 \\
\hline Peri-lesion emphysema, n (\%) & $11(16.7)$ & $9(20.5)$ & $14(28.6)$ & 0.301 & $5(29.4)$ & $2(18.2)$ & $0(0.0)$ & $0.048^{*}$ \\
\hline Air bronchogram, n (\%) & $20(30.3)$ & $20(45.5)$ & $35(71.4)$ & $0.000^{*}$ & $5(29.4)$ & $8(72.7)$ & $9(75.0)$ & $0.018^{*}$ \\
\hline Bubble-like lucency, n (\%) & $12(18.2)$ & $9(20.5)$ & $11(22.5)$ & 0.851 & $1(5.9)$ & $3(27.3)$ & $4(33.3)$ & 0.119 \\
\hline Lobulation, n (\%) & $59(89.4)$ & $41(93.2)$ & $44(89.8)$ & 0.770 & $13(76.5)$ & $10(90.9)$ & $9(75.0)$ & 0.526 \\
\hline Spiculation, n (\%) & $64(97.0)$ & $44(100.0)$ & 45 (91.8) & 0.065 & $17(100.0)$ & $9(81.8)$ & $10(83.3)$ & 0.092 \\
\hline Pleural tag, n (\%) & $59(89.4)$ & $41(93.2)$ & $45(91.8)$ & 0.775 & $11(64.7)$ & $8(72.7)$ & 7 (58.3) & 0.767 \\
\hline Vascular convergence, $\mathrm{n}(\%)$ & $32(48.5)$ & $25(56.8)$ & $42(85.7)$ & $0.000^{*}$ & $14(82.4)$ & $9(81.8)$ & $5(41.7)$ & $0.043^{*}$ \\
\hline Vascular involvement, n (\%) & $19(28.8)$ & $10(22.7)$ & $5(10.2)$ & 0.054 & $6(35.3)$ & $5(45.5)$ & $2(16.7)$ & 0.301 \\
\hline $\begin{array}{l}\text { Homogenous attenuation, } \mathrm{n} \\
(\%)\end{array}$ & 49 (74.2) & $31(70.5)$ & 45 (91.8) & $0.022^{\star}$ & $12(70.6)$ & 9 (81.8) & $3(25.0)$ & $0.009^{*}$ \\
\hline Pleural effusion, n (\%) & $22(33.3)$ & $17(38.6)$ & 21 (42.9) & 0.575 & 7 (41.2) & 3 (27.3) & 4 (33.3) & 0.743 \\
\hline
\end{tabular}

*, P value <0.05. CT, computed tomography; LUAD, lung adenocarcinomas; EGFR, epidermal growth factor receptor; RUL, right upper lobe; $\mathrm{RML}$, right middle lobe; RLL, right lower lobe; LUL, left upper lobe; LLL, left lower lobe; GGO, ground-glass opacity; IQR, interquartile range. 


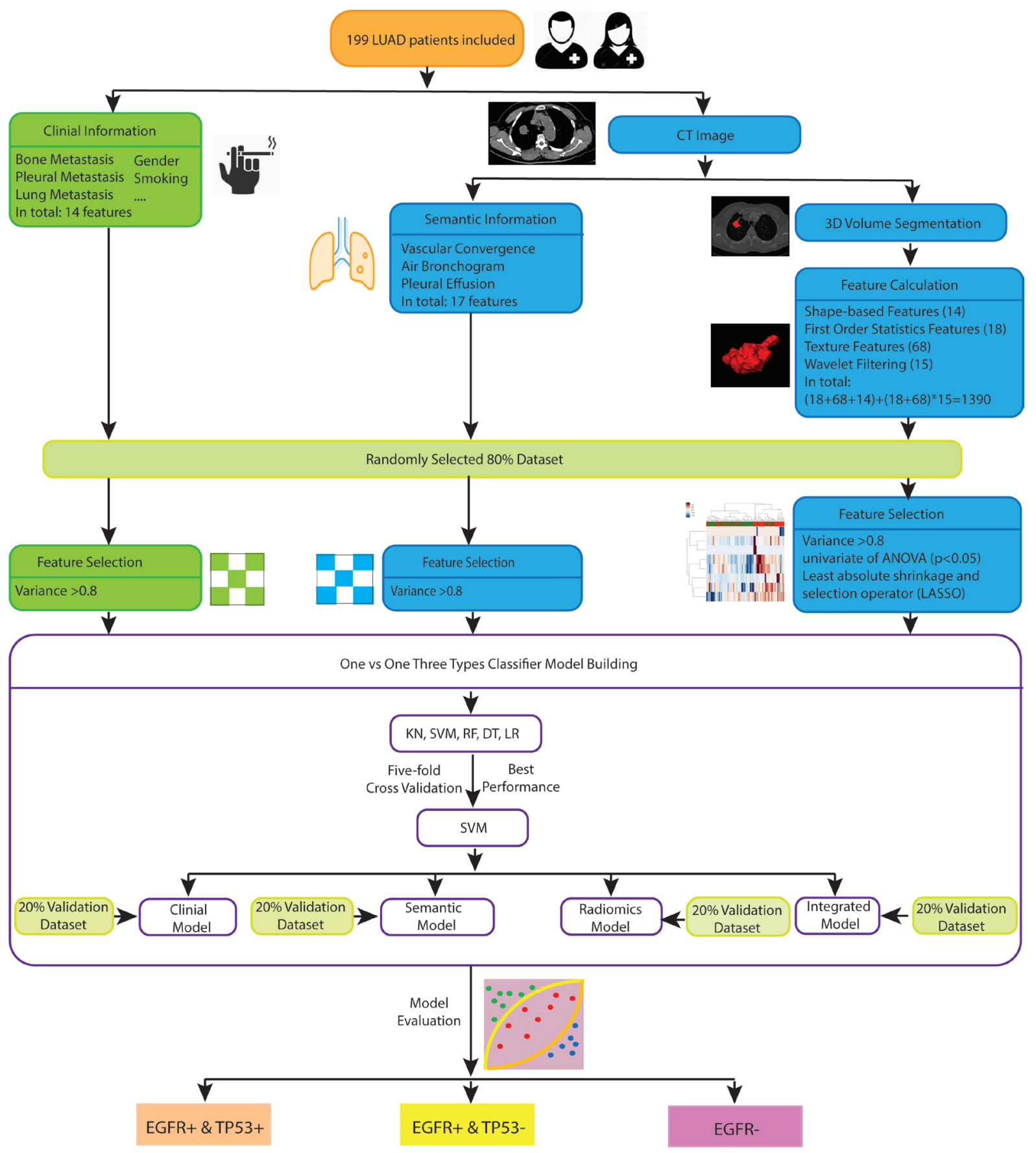

Figure 2 The flowchart of feature selection and model building. LUAD, lung adenocarcinomas; CT, computed tomography; EGFR, epidermal growth factor receptor; KN, k-nearest neighbors; SVM, support vector machine; RF, random forest; DT, decision tree; LR, logistic regression. 
by integrated model.

\section{Feature selection}

Feature selection was performed separately for each type of features. Three steps were applied to reduce dimensionality: (I) features with variance larger than 0.8 were included for further analysis; (II) univariate feature selection is done by ANOVA (continuous variable) or chi-square test (discrete variable) to explore the associations between features and genotype. The features with $\mathrm{P}$ value above 0.05 were excluded from further analysis; (III) the most significant features were selected by the least absolute shrinkage and selection operator (LASSO) method, which is very effective to reduce dimensionality. For clinical model and semantic model, since the features number before selection was small, only step one was applied for feature reduction. For radiomics model and integrated model, all three steps were executed, and the nonzero feature coefficients ranking the first five were selected for each binary classifier to avoid overfitting.

\section{Statistical analysis}

Statistical analyses were performed by using SPSS 22.0 (IBM, USA). Continuous variables were expressed as median [interquartile range (IQR)]. Categorical variables were displayed as frequency, $\mathrm{n}(\%)$. All machine learning analyses were performed using the Python package scikitlearn (0.19.0), and statistical plots were generated by Matplotlib (2.0.2). Area under the receiver-operating characteristic curves (AUCs) were calculated to evaluate the binary classifiers, and the best ones were applied for the final result. Statistical metrics, including accuracy, sensitivity, specificity, precision, recall, F1 score, were also calculated to evaluate the overall performance of the multiclass classifier. These statistical metrics for multiclass classification were defined similar as those for binary classification. It should be noted that once we picked up one category as positive, the other two are automatically negative. The Youden Index was used to generate the optimal threshold to convert probabilities into binarized labels.

\section{Results}

\section{Basic clinical and semantic CT imaging characteristics}

A total of 199 patients were included in our study, including
$66(41.5 \%)$ cases of $E G F R^{-}, 44(27.7 \%)$ cases of $E G F R^{+}$\& $T P 53^{+}, 49(30.8 \%)$ cases of $E G F R^{+} \& T P 53^{-}$in the training cohort and 17 (42.5\%) cases of EGFR, $11(27.5 \%)$ cases of $E G F R^{+} \& T P 53^{+}, 12(30.0 \%)$ cases of $E G F R^{+} \& T P 53^{-}$in the validation cohort. Patient clinicopathological characteristics in the training and validation cohorts are given in Table 1. Gender, smoking status, bone metastasis, lung metastasis, pleural metastasis shows significant differences between the three groups both in the training and validation cohorts, with $\mathrm{P}$ value $<0.05$ respectively.

For the three groups of $E G F R^{-}, E G F R^{+} \& T P 53^{+}$, $E G F R^{+} \& T P 53^{-}$, the detailed information of semantic CT imaging characteristics before treatment in the training and validation cohorts are given in Table 2. The differences in air bronchogram, vascular convergence, homogenous attenuation between the three groups both in the training and validation cohorts are significant. Presence of GGO shows significant differences in the training cohort and peri-lesion emphysema shows significant differences in the validation cohort.

\section{Feature selection and the performance of base binary classifiers}

The selected features for the clinical, semantic, radiomics, and integrated models were shown in Table 3. Each group contained three different base binary classifiers used in multiclass classifier.

Algorithms of SVM, KN, RF, and LR were applied to build base binary classifiers using selected features from the training cohort, and their performance were compared. We selected the SVM algorithm with the best performance for the training dataset as shown in Table 4, and all the analysis and validation were based on it. The Radial basis function (RBF) kernel, also called the RBF kernel, were utilized in the SVM algorithm, and the key hyperparameters gamma and $\mathrm{C}$ values were shown in Table S1. For the SVM model, the results indicated that the AUCs were $0.731,0.653,0.843$ for the three base binary classifiers in the clinical model, and the values were $0.792,0.613,0.781$ for the semantic model. The radiomics model yielded relatively higher efficacy, with AUCs of 0.785, 0.771, and 0.812, compared with the former two models. When integrating all the information, the performance of the model was improved, with AUCs of $0.831,0.767$, and 0.892 . Moreover, it revealed that it would be possible to differentiate precisely $\left(E G F R^{+} \& T P 53^{-}\right)$from $\left(E G F R^{-}\right)$, with $>0.780$ AUC across all models. Moderate performance was achieved in differentiating $\left(E G F R^{+} \&\right.$ 
Table 3 Feature selection for the four different models

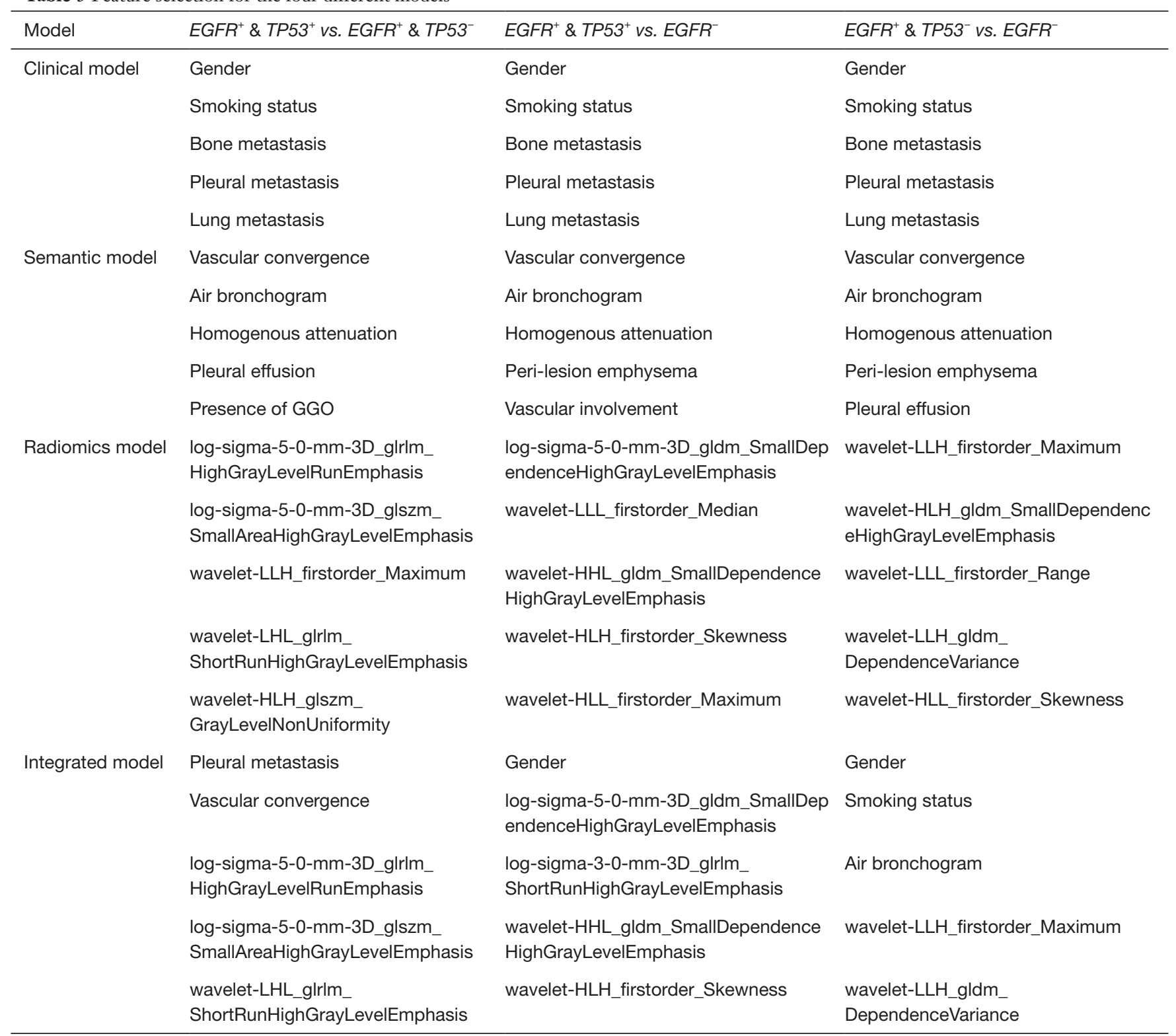

GGO, ground-glass opacity.

$\left.T P 53^{+}\right)$from $\left(E G F R^{+} \& T P 53^{-}\right),\left(E G F R^{+} \& T P 53^{+}\right)$from $\left(E G F R^{-}\right)$, except the poor accuracy was achieved when discriminating $\left(E G F R^{+} \& T P 53^{+}\right)$from $\left(E G F R^{-}\right)$using the clinical (AUC, $0.653 \pm 0.112$ ) and semantic model (AUC, $0.613 \pm 0.057)$.

\section{Multiclass classification strategy}

The performance of the multiclass classification for the validation dataset, in our study was three-type classification, was shown in Table 5 and Figure 3. When AUCs were used to evaluate the distinguishing efficacy of the models, the integrated model displayed the best performance for all the three subtypes of co-mutations: $E G F R^{-}$(AUC, 0.857; accuracy, 0.817; sensitivity, 0.998; specificity, 0.663), EGFR \& $T P 53^{+}$(AUC, 0.791; accuracy, 0.758; sensitivity, 0.762; specificity, 0.783), EGFR \& TP53- (AUC, 0.761; accuracy, 0.813; sensitivity, 0.594; specificity, 0.977). Although the 
Table 4 Comparison of the AUCs obtained from base binary classifiers using the different algorithms among the four models

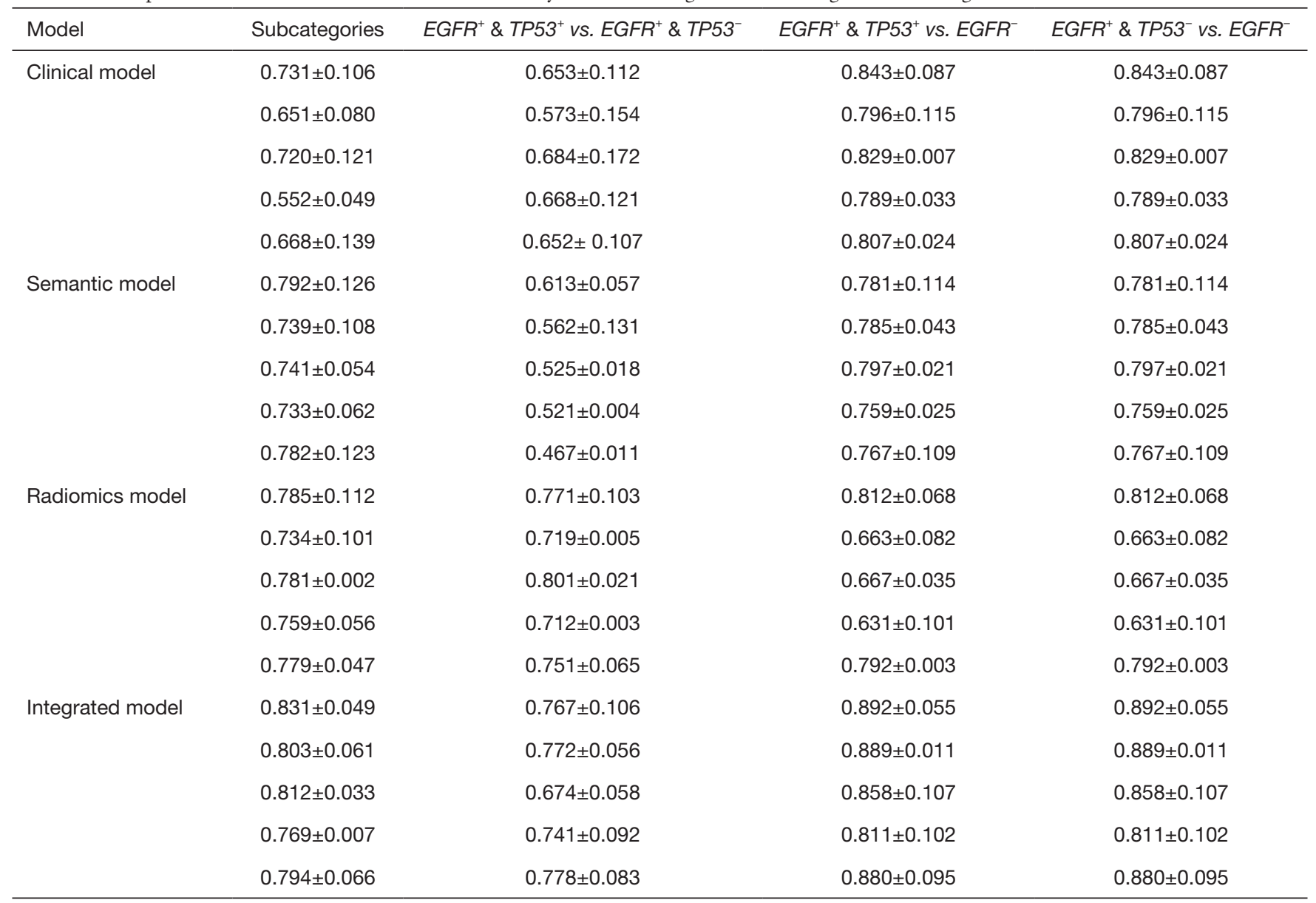

EGFR, epidermal growth factor receptor; AUC, area under the receiver-operating characteristic curve, expressed by mean \pm standard deviation; SVM, support vector machine; KN, k-nearest neighbors; RF, random forest; DT, decision tree; LR, logistic regression.

Table 5 Comparison of the final performance of multiclass classifiers among the four different models

\begin{tabular}{lcccccccc}
\hline Model & Subcategories & Accuracy & Sensitivity & Specificity & Precision & Recall & F1 score & AUC \\
\hline Clinical model & $E G F R^{+} \& T P 53^{+}$ & 0.501 & 0.765 & 0.477 & 0.314 & 0.781 & 0.433 & 0.483 \\
& $E G F R^{+} \& T P 53^{-}$ & 0.723 & 0.809 & 0.653 & 0.534 & 0.806 & 0.621 & 0.685 \\
& $E G F R^{-}$ & 0.617 & 0.813 & 0.521 & 0.538 & 0.814 & 0.652 & 0.659 \\
Semantic model & $E G F R^{+} \& T P 53^{+}$ & 0.406 & 0.891 & 0.207 & 0.354 & 0.895 & 0.427 & 0.301 \\
& $E G F R^{+} \& T P 53^{-}$ & 0.502 & 0.899 & 0.273 & 0.368 & 0.901 & 0.547 & 0.461 \\
& $E G F R^{-}$ & 0.606 & 0.772 & 0.513 & 0.562 & 0.798 & 0.611 & 0.609 \\
Radiomics & $E G F R^{+} \& T P 53^{+}$ & 0.842 & 0.528 & 0.957 & 0.816 & 0.571 & 0.693 & 0.762 \\
model & $E G F R^{+} \& T P 53^{-}$ & 0.773 & 0.829 & 0.687 & 0.594 & 0.802 & 0.681 & 0.753 \\
& $E G F R^{-}$ & 0.796 & 0.728 & 0.816 & 0.792 & 0.727 & 0.765 & 0.836 \\
Integrated & $E G F R^{+} \& T P 53^{+}$ & 0.758 & 0.762 & 0.783 & 0.569 & 0.791 & 0.672 & 0.791 \\
model & $E G F R^{+} \& T P 53^{-}$ & 0.813 & 0.594 & 0.977 & 0.782 & 0.592 & 0.674 & 0.761 \\
& $E G F R^{-}$ & 0.817 & 0.998 & 0.663 & 0.694 & 0.989 & 0.831 & 0.857 \\
\hline
\end{tabular}

EGFR, epidermal growth factor receptor; AUC, area under the receiver-operating characteristic curve. 
A

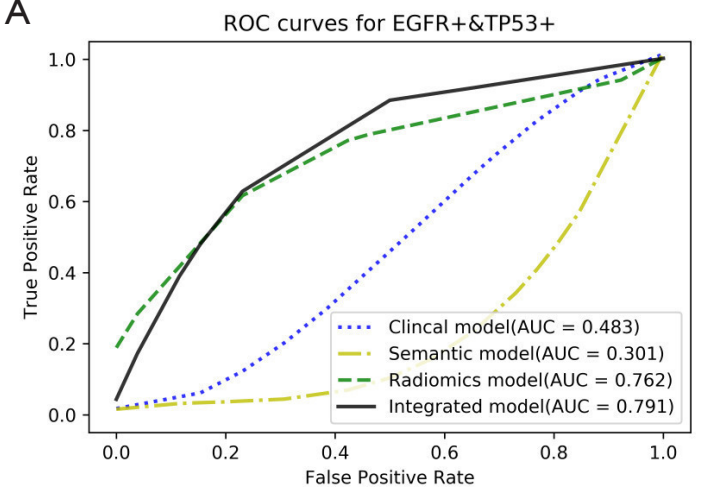

C

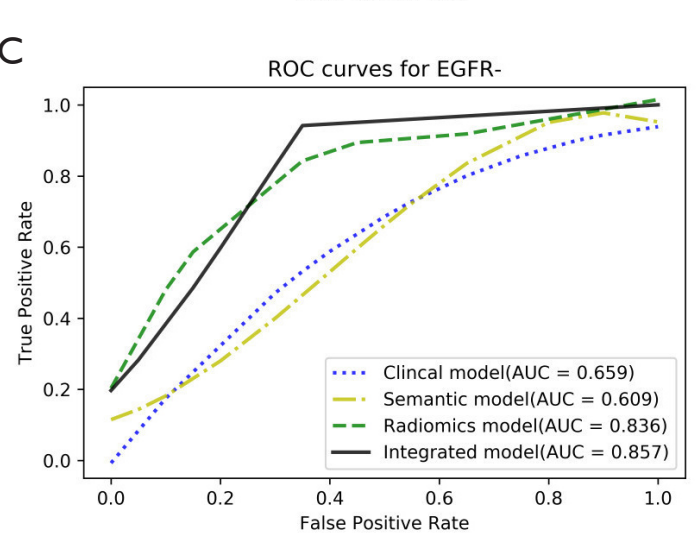

B

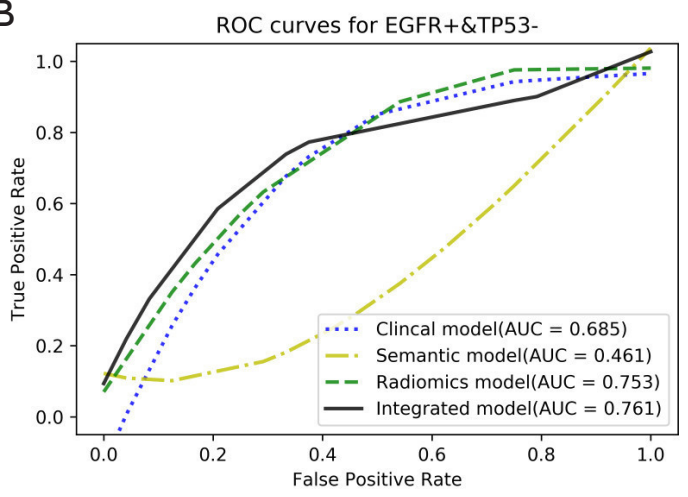

Figure 3 The performance of the three-type classifier. (A) ROC curves of the four different models for distinguishing the co-mutation status of $E G F R^{+} \& T P 53^{+}$. (B) ROC curves of the four different models for distinguishing the co-mutation status of $E G F R^{+} \& T P 53^{-}$. (C) ROC curves of the four different models for distinguishing the co-mutation status of $E G F R^{-}$. Area under the ROC curves (AUCs) were used to evaluate the distinguishing efficacy of the four different models. The integrated model displayed the best performance for all the three subtypes of co-mutation status. ROC, receiver-operating characteristic; EGFR, epidermal growth factor receptor.

integrated model showed the best prediction efficiency, the radiomics model showed only a slight decrease for all the three subtypes compared with it: $E G F R^{-}$(AUC, 0.836; accuracy, 0.796; sensitivity, 0.728; specificity, 0.816), $E_{G F R^{+}} \& T_{P 53^{+}}$(AUC, 0.762; accuracy, 0.842; sensitivity, 0.528; specificity, 0.957), EGFR \& TP53- (AUC, 0.753; accuracy, 0.773; sensitivity, 0.829; specificity, 0.687). The clinical model and the semantic model showed unsatisfied distinguishing efficiency, with AUCs less than 0.700 for all the three subtypes.

\section{Discussion}

In the present study, we developed and validated a multiclass classification strategy for the pretherapeutic individualized prediction of primary overlapping mutations involving TP53 and EGFR in advanced LUAD. The integrated model is promising to distinguish $E G F R^{+}$\& $T_{P 53^{+}}, E G F R^{+} \& T P 53^{-}, E G F R^{-}$, with AUC more than 0.750 . According to recent studies, in EGFR-mutated NSCLC patients treated with TKIs, EGFR co-mutated with TP53 could reduce responsiveness to TKIs and worsen patients' prognosis compared to TP53 wild type patients $(12,13)$. Identify mutation types in different combinations can help to select best responders to target therapy. The model built by our team has the potential to preselect patients who will be sensitive to TKI therapy and patients who may have a better clinical outcome. Our study provided an alternative way to non-invasively assess TP53 genotype combined with $E G F R$, offered a great supplement to biopsy. To the best of our knowledge, this is the first study to predict overlapping mutations regarding TP53 based on CT images, it may serve as an alternative marker to select the best responders to TKIs in EGFR-mutated LUAD. 
So far, there have been many studies reported the correlation between imaging features and somatic mutations (22-31) as well as molecular expression (32). Image as a non-invasive method has great potency in predicting genotype and molecular for many kinds of tumors, it is likely to become an important alternative marker for treatment decision making. For lung cancer, most previous studies focus on the predictive value of CT features on EGFR mutation (22-27), and achieved favourable outcome, with AUC more than 0.800 (22). The established models may be helpful in selecting patients who will be adaptable to TKIs prior to treatment. Considering the potential impact of TP53 mutation on the therapeutic effect of TKI, further attention should be paid to the co-mutation status of TP53. We try to apply the multiclass classification strategy to solve the problem of overlapping mutations involving EGFR and TP53. The model proposed by our team can predict of the specific three mutational status by one step. Therefore, our model extends the potential application value of the existed model, it can better help to select the best responders to TKI therapy.

Several studies have explored the correlation between imaging features and TP53 mutation in some kinds of cancers, including LUAD (33), pancreatic ductal adenocarcinoma $(34,35)$, head and neck cancer (36), colorectal cancer (37), glioma (38). In the study conducted by Wang et al. (33), they enrolled 51 patients with resectable early stage LUAD. The radiomics signature yielded a median AUC value of 0.604 , and 0.586 respectively in predicting EGFR and TP53 mutations. The combined radiomics and clinical model further improved the prediction performance, with AUC 0.697 for EGFR mutation, and 0.656 for TP53 mutation, respectively. Different from their study, we focused on advanced LUAD which may need TKI therapy if a sensitive gene mutation is detected. As for TP53, we studied the overlapping mutation status of EGFR and TP53, instead of predicting the mutation status of EGFR and TP53 alone. Despite the concurrent TP53 genomic alteration in EGFR mutant LUAD demonstrated distinctive therapeutic responses to TKIs, so far there is no relevant predicting biomarkers to distinguish this co-mutation condition. The multiclass classification radiomics model proposed by our team may be an auxiliary in screening the best responder to TKIs in advanced lung cancer.

In this study, the proposed multiclass classification radiomics model provides potential clinical utility from the following perspectives. (I) The proposed radiomics model can be used as an alternative tool to noninvasively predict TP53 genotype easily through routine CT images which is necessary for lung cancer patients to perform pretherapeutic staging, without adding cost. (II) As well studied by other researchers that CT-based imaging features can predict EGFR genotype (19,39-42), thus further distinguish TP53 genotype at the same time becomes feasible. This is promising for patients who are unable to afford large panel sequencing due to poor economic conditions. (III) For patients who have poor response to TKI therapy during treatment and are unwilling to conduct re-biopsy for repetitive sequencing or unable to acquire enough tumor tissue for re-biopsy, the proposed model is an alternative for treatment decision making. This approach makes dynamic molecular diagnosis and timely adjustment of treatment possible. (IV) Although there is no targeted drug for TP53 co-mutation at present, with the rapid development of treatment modalities for advanced lung cancer, there may be some new therapeutic regimens for the clinical condition of TP53 overlapping mutation in the future to improve patient prognosis.

Despite the encouraging and promising findings of our present study, it still has several limitations. First, our findings deserve further study with expanded samples and extra external validation. A large-scale study enrolling more patients may definitely help validate and improve its applicability as an effective prediction tool for predicting TP53 genotype in treatment decision making for LUAD. Second, our study focused on LUAD and did not address other histologic subtypes. Third, despite the present study was a multicenter study, the results cannot be generalized to other populations because gene mutation rate can be affected by race. Fourth, a large number of the gene sequencing results in this study were based on fine needle aspiration biopsy, so tumor heterogeneity is inevitable.

In conclusion, the proposed radiomics model is expected to distinguish co-mutation status involving TP53 and EGFR. It may have a potential application value in preselecting patients who will be adaptable to and sensitive to TKIs and have better prognosis.

\section{Acknowledgments}

Funding: None.

\section{Footnote}

Reporting Checklist: The authors have completed the STARD 
reporting checklist. Available at http://dx.doi.org/10.21037/ atm-20-6473

Data Sharing Statement: Available at http://dx.doi. org/10.21037/atm-20-6473

Peer Review File: Available at http://dx.doi.org/10.21037/ atm-20-6473

Conflicts of Interest: All authors have completed the ICMJE uniform disclosure form (available at http://dx.doi. org/10.21037/atm-20-6473). The authors have no conflicts of interest to declare.

Ethical Statement: The authors are accountable for all aspects of the work in ensuring that questions related to the accuracy or integrity of any part of the work are appropriately investigated and resolved. This study conformed to the provisions of the Declaration of Helsinki (as revised in 2013). This study was approved by the institutional ethics committee of the First Affiliated Hospital of Sun Yat-sen University \{No.[2013]C-084\}. Informed consent was waived.

Open Access Statement: This is an Open Access article distributed in accordance with the Creative Commons Attribution-NonCommercial-NoDerivs 4.0 International License (CC BY-NC-ND 4.0), which permits the noncommercial replication and distribution of the article with the strict proviso that no changes or edits are made and the original work is properly cited (including links to both the formal publication through the relevant DOI and the license). See: https://creativecommons.org/licenses/by-nc-nd/4.0/.

\section{References}

1. Bray F, Ferlay J, Soerjomataram I, et al. Global cancer statistics 2018: GLOBOCAN estimates of incidence and mortality worldwide for 36 cancers in 185 countries. CA Cancer J Clin 2018;68:394-424.

2. Zhou F, Zhou C. Lung cancer in never smokers-the East Asian experience. Transl Lung Cancer Res 2018;7:450-63.

3. Jemal A, Miller KD, Ma J, et al. Higher Lung Cancer Incidence in Young Women Than Young Men in the United States. N Engl J Med 2018;378:1999-2009.

4. Chen YJ, Roumeliotis TI, Chang YH, et al. Proteogenomics of Non-smoking Lung Cancer in East Asia Delineates Molecular Signatures of Pathogenesis and
Progression. Cell 2020;182:226-244.e17.

5. Wu FZ, Huang YL, Wu CC, et al. Assessment of Selection Criteria for Low-Dose Lung Screening CT Among Asian Ethnic Groups in Taiwan: From Mass Screening to Specific Risk-Based Screening for Non-Smoker Lung Cancer. Clin Lung Cancer 2016;17:e45-56.

6. Yang CY, Yang JC, Yang PC. Precision Management of Advanced Non-Small Cell Lung Cancer. Annu Rev Med 2020;71:117-36.

7. Shi $\mathrm{Y}, \mathrm{Au} \mathrm{JS}$, Thongprasert $\mathrm{S}$, et al. A prospective, molecular epidemiology study of EGFR mutations in Asian patients with advanced non-small-cell lung cancer of adenocarcinoma histology (PIONEER). J Thorac Oncol 2014;9:154-62.

8. Rosell R, Carcereny E, Gervais R, et al. Erlotinib versus standard chemotherapy as first-line treatment for European patients with advanced EGFR mutation-positive non-small-cell lung cancer (EURTAC): a multicentre, open-label, randomised phase 3 trial. Lancet Oncol 2012;13:239-46.

9. Arbour KC, Riely GJ. Systemic Therapy for Locally Advanced and Metastatic Non-Small Cell Lung Cancer: A Review. JAMA 2019;322:764-74.

10. Russo A, Franchina T, Ricciardi GR, et al. A decade of EGFR inhibition in EGFR-mutated non small cell lung cancer (NSCLC): Old successes and future perspectives. Oncotarget 2015;6:26814-25.

11. Maemondo M, Inoue A, Kobayashi K, et al. Gefitinib or chemotherapy for non-small-cell lung cancer with mutated EGFR. N Engl J Med 2010;362:2380-8.

12. Canale M, Petracci E, Delmonte A, et al. Impact of TP53 Mutations on Outcome in EGFR-Mutated Patients Treated with First-Line Tyrosine Kinase Inhibitors. Clin Cancer Res 2017;23:2195-202.

13. Jiao XD, Qin BD, You P, et al. The prognostic value of TP53 and its correlation with EGFR mutation in advanced non-small cell lung cancer, an analysis based on cBioPortal data base. Lung Cancer 2018;123:70-5.

14. Deben C, Deschoolmeester V, Lardon F, et al. TP53 and MDM2 genetic alterations in non-small cell lung cancer: Evaluating their prognostic and predictive value. Crit Rev Oncol Hematol 2016;99:63-73.

15. Ma X, Le Teuff G, Lacas B, et al. Prognostic and Predictive Effect of TP53 Mutations in Patients with Non-Small Cell Lung Cancer from Adjuvant CisplatinBased Therapy Randomized Trials: A LACE-Bio Pooled Analysis. J Thorac Oncol 2016;11:850-61.

16. Schwaederlé M, Lazar V, Validire P, et al. VEGF-A 
Expression Correlates with TP53 Mutations in Non-Small Cell Lung Cancer: Implications for Antiangiogenesis Therapy. Cancer Res 2015;75:1187-90.

17. Zhou M, Leung A, Echegaray S, et al. Non-Small Cell Lung Cancer Radiogenomics Map Identifies Relationships between Molecular and Imaging Phenotypes with Prognostic Implications. Radiology 2018;286:307-15.

18. Gevaert O, Xu J, Hoang CD, et al. Non-small cell lung cancer: identifying prognostic imaging biomarkers by leveraging public gene expression microarray data--methods and preliminary results. Radiology 2012;264:387-96.

19. Wang S, Shi J, Ye Z, et al. Predicting EGFR mutation status in lung adenocarcinoma on computed tomography image using deep learning. Eur Respir J 2019;53:1800986.

20. Yang X, Dong X, Wang J, et al. Computed TomographyBased Radiomics Signature: A Potential Indicator of Epidermal Growth Factor Receptor Mutation in Pulmonary Adenocarcinoma Appearing as a Subsolid Nodule. Oncologist 2019;24:e1156-64.

21. Li Y, Lu L, Xiao M, et al. CT Slice Thickness and Convolution Kernel Affect Performance of a Radiomic Model for Predicting EGFR Status in Non-Small Cell Lung Cancer: A Preliminary Study. Sci Rep 2018;8:17913.

22. Jia TY, Xiong JF, Li XY, et al. Identifying EGFR mutations in lung adenocarcinoma by noninvasive imaging using radiomics features and random forest modeling. Eur Radiol 2019;29:4742-50.

23. Mei D, Luo Y, Wang Y, et al. CT texture analysis of lung adenocarcinoma: can Radiomic features be surrogate biomarkers for EGFR mutation statuses. Cancer Imaging 2018; 18:52.

24. Choi CM, Kim MY, Lee JC, et al. Advanced lung adenocarcinoma harboring a mutation of the epidermal growth factor receptor: CT findings after tyrosine kinase inhibitor therapy. Radiology 2014;270:574-82.

25. Hasegawa M, Sakai F, Ishikawa R, et al. CT Features of Epidermal Growth Factor Receptor-Mutated Adenocarcinoma of the Lung: Comparison with Nonmutated Adenocarcinoma. J Thorac Oncol 2016;11:819-26.

26. Togashi Y, Masago K, Kubo T, et al. Association of diffuse, random pulmonary metastases, including miliary metastases, with epidermal growth factor receptor mutations in lung adenocarcinoma. Cancer 2011;117:819-25.

27. Hsu JS, Huang MS, Chen CY, et al. Correlation between EGFR mutation status and computed tomography features in patients with advanced pulmonary adenocarcinoma. J
Thorac Imaging 2014;29:357-63.

28. Grossmann P, Stringfield O, El-Hachem N, et al. Defining the biological basis of radiomic phenotypes in lung cancer. Elife 2017;6:e23421.

29. Podolsky MD, Barchuk AA, Kuznetcov VI, et al. Evaluation of Machine Learning Algorithm Utilization for Lung Cancer Classification Based on Gene Expression Levels. Asian Pac J Cancer Prev 2016;17:835-8.

30. Miles KA, Ganeshan B, Rodriguez-Justo M, et al. Multifunctional imaging signature for V-KI-RAS2 Kirsten rat sarcoma viral oncogene homolog (KRAS) mutations in colorectal cancer. J Nucl Med 2014;55:386-91.

31. Yamamoto S, Korn RL, Oklu R, et al. ALK molecular phenotype in non-small cell lung cancer: CT radiogenomic characterization. Radiology 2014;272:568-76.

32. Zhu Y, Liu YL, Feng Y, et al. A CT-derived deep neural network predicts for programmed death ligand-1 expression status in advanced lung adenocarcinomas. Ann Transl Med 2020;8:930.

33. Wang X, Kong C, Xu W, et al. Decoding tumor mutation burden and driver mutations in early stage lung adenocarcinoma using CT-based radiomics signature. Thorac Cancer 2019;10:1904-12.

34. Lim CH, Cho YS, Choi JY, et al. Imaging phenotype using F-fluorodeoxyglucose positron emission tomographybased radiomics and genetic alterations of pancreatic ductal adenocarcinoma. Eur J Nucl Med Mol Imaging 2020;47:2113-22.

35. Attiyeh MA, Chakraborty J, McIntyre CA, et al. CT radiomics associations with genotype and stromal content in pancreatic ductal adenocarcinoma. Abdom Radiol (NY) 2019;44:3148-57.

36. Zwirner K, Hilke FJ, Demidov G, et al. Radiogenomics in head and neck cancer: correlation of radiomic heterogeneity and somatic mutations in TP53, FAT1 and KMT2D. Strahlenther Onkol 2019;195:771-9.

37. Chen SW, Shen WC, Chen WT, et al. Metabolic Imaging Phenotype Using Radiomics of [F]FDG PET/CT Associated with Genetic Alterations of Colorectal Cancer. Mol Imaging Biol 2019;21:183-90.

38. Zhang X, Tian Q, Wang L, et al. Radiomics Strategy for Molecular Subtype Stratification of Lower-Grade Glioma: Detecting IDH and TP53 Mutations Based on Multimodal MRI. J Magn Reson Imaging 2018;48:916-26.

39. Girard N, Sima CS, Jackman DM, et al. Nomogram to predict the presence of EGFR activating mutation in lung adenocarcinoma. Eur Respir J 2012;39:366-72.

40. Liu Y, Kim J, Qu F, et al. CT Features Associated with 
Epidermal Growth Factor Receptor Mutation Status in Patients with Lung Adenocarcinoma. Radiology 2016;280:271-80.

41. Yano M, Sasaki H, Kobayashi Y, et al. Epidermal growth factor receptor gene mutation and computed tomographic findings in peripheral pulmonary adenocarcinoma. J

Cite this article as: Zhu Y, Guo YB, Xu D, Zhang J, Liu ZG, Wu X, Yang XY, Chang DD, Xu M, Yan J, Ke ZF, Feng ST, Liu YL. A computed tomography (CT)-derived radiomics approach for predicting primary co-mutations involving TP53 and epidermal growth factor receptor $(E G F R)$ in patients with advanced lung adenocarcinomas (LUAD). Ann Transl Med 2021;9(7):545. doi: 10.21037/atm-20-6473
Thorac Oncol 2006;1:413-6.

42. Zhou JY, Zheng J, Yu ZF, et al. Comparative analysis of clinicoradiologic characteristics of lung adenocarcinomas with ALK rearrangements or EGFR mutations. Eur Radiol 2015;25:1257-66. 
Supplementary

Table S1 Hyperparameters of SVM for base binary classifiers among the four models

\begin{tabular}{|c|c|c|c|c|c|c|}
\hline Model & \multicolumn{2}{|c|}{$E G F R^{+} \& T P 53^{+}$vs. EGFR ${ }^{+} \& T P 53^{-}$} & \multicolumn{2}{|c|}{$E G F R^{+} \& T P 53^{+}$vs. EGFR } & \multicolumn{2}{|c|}{$E G F R^{+} \& T P 53^{-}$vs. EGFR } \\
\hline Clinical model & 0.0001 & 1 & 0.001 & 100 & 0.0001 & 100 \\
\hline Semantic model & 0.01 & 10 & 0.0001 & 10 & 0.01 & 100 \\
\hline Radiomics model & 0.0001 & 100 & 0.1 & 100 & 0.0001 & 100 \\
\hline
\end{tabular}

EGFR, epidermal growth factor receptor; SVM, support vector machine. 